
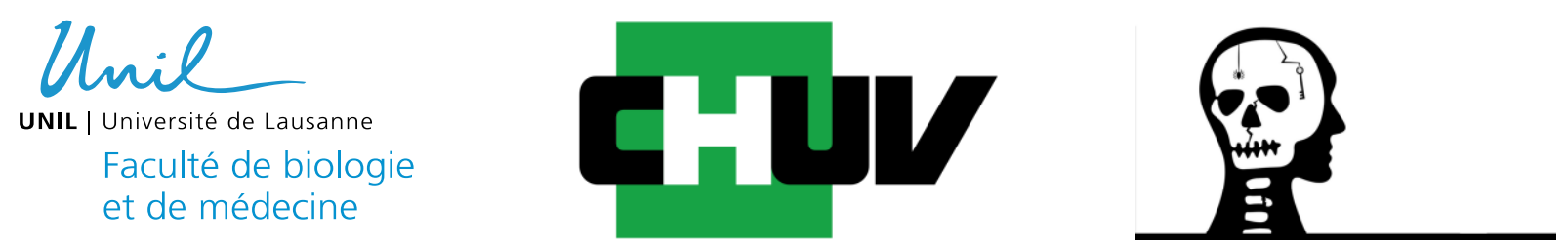

Mémoire de Maîtrise en médecine No 5576

\title{
Biomarkers of cerebral damage in fatal hypothermia
}

\author{
Etudiant \\ Morleo Bastien \\ Tuteur \\ Palmiere Cristian \\ Centre Universitaire Romand de Médecine Légale \\ Expert \\ Grouzmann Eric \\ Département des laboratoires
}

Lausanne, 09.03.2019 
Bastien Morleo, Gregor Teresinski, Guillaume Rousseau, Rexson Tse, Camilla Tettamanti, Marc Augsburger, Cristian Palmiere

Biomarkers of cerebral damage in fatal hypothermia: preliminary results.

\section{Abstract}

The identification of hypothermia as the cause of death remains challenging in forensic pathology because of unspecific radiological, morphological, and biochemical results. Hyperaemia, oedema, and petechial haemorrhages within the cerebral parenchyma were described in cases of death by hypothermia. On the other hand, the effect of low temperatures in the brain has been speculated to cause local injuries on a cellular level with potential occurrences of necrosis, and inflammation. In the study herein described, endocan, alkaline phosphatase, neuronal specific enolase, S100 protein subunit B, glial fibrillary acidic protein, and C-reactive protein were measured in postmortem serum from femoral blood and cerebrospinal fluid in a series of hypothermia fatalities and control cases. The combination of data collected failed to identify a specific biochemical profile for death by hypothermia in postmortem serum and/or the cerebrospinal fluid, thus suggesting that an alternative panel of brain damage biomarkers indicative of diffuse hypoxic brain injury need to be defined in hypothermia fatalities.

Keywords: Hypothermia; Postmortem biochemistry; Biomarkers; Autopsy.

Introduction

Hypothermia is a condition characterized by a decrease in body temperature to below 35 degrees and occurs when the loss of heat within an organism is greater than its production (1).

Potentially fatal hypothermia develops insidiously and compromises numerous physiological functions, including neuromuscular, cardiovascular, and respiratory functions. The onset of hypothermia is typically accompanied by neurological symptoms such as hampered speech, lethargy, potential convulsions, ataxia, loss of coordination, and other changes including drowsiness, confusion, and unconsciousness (usually when body temperature has dropped below 30 degrees), which can further progress towards coma and death (2-4).

In the realm of forensic pathology, injuries such as cerebral hyperaemia, cerebral oedema, and petechial haemorrhages, or so called cold-induced purpura, have been described in some cases of death by hypothermia, for instance in the area of the third ventricle. Moreover, haemorrhagic suffusions of the cerebral parenchyma have been observed by a few authors in cases of fatal hypothermia and have been associated with the increase in blood pressure in the cerebral arteries due to enhanced catecholamine secretion caused by cold exposure (5).

Based on both the characteristics of the neurologic clinical picture of developing hypothermia and the postmortem morphological changes described over the course of time by various authors in cases of lethal hypothermia, the question arises as to whether the establishment of the (lethal) hypothermic process, from the initial phases to the most advanced ones, could result in neuro-morphological and neuro-biochemical changes, the latter analysable through targeted laboratory analyses in postmortem serum and cerebrospinal fluid (CSF).

Hence, the aim of this study was to measure the concentrations of a series of molecules contained in (or expressed by) vascular endothelial cells, glial cells, and neuronal cells in physiological conditions in biological fluids collected at autopsy (postmortem serum from femoral blood and CSF) in a series of hypothermia fatalities and control cases, in order to assess the possible usefulness of their measurement for the postmortem diagnosis of antemortem cold exposure.

These molecules included endocan, alkaline phosphatase (ALP), neuronal specific enolase (NSE), S100 protein subunit B (S100B), glial fibrillary acidic protein (GFAP), and C-reactive protein (CRP). 
Some of these molecules have already been topics of study in the clinical setting in situations of neurological interest (traumatic brain injuries, brain tumours, strokes, etc.), as well as in the forensic setting in the postmortem serum and/or CSF in various circumstances of forensic interest (i.e. traumatic brain injury), but never in studies specific and exclusive to cases of lethal hypothermia (627).

Materials and methods

Study design and study populations

The present study was performed during the period 2016-2018. A total of 60 forensic autopsy cases (42 males and 18 females) with a mean age of 51.2 years (range 21-79) were selected and two study groups (hypothermia cases and control cases) prospectively identified. All cases included in the study originated from forensic pathology practice and underwent forensic investigations as requested by local inquiring authorities.

The first study group consisted of 30 hypothermia fatalities ( 24 males and 6 females).

The postmortem intervals for these cases was defined as the time elapsed between body discovery and specimen collection and ranged from $4 \mathrm{~h}$ to $72 \mathrm{~h}$. The most relevant inclusion criteria consisted of:

- hypothermia as the cause of death,

- postmortem serum and CSF availability at autopsy,

- exclusion of traumatic brain injury as the cause of death (exclusion of intracranial and intracerebral bleeding, exclusion of cortical contusion, exclusion of cortical and cerebral tissue damage).

The cause of death was attributed to hypothermia (death following cold exposure) based on these inclusion and exclusion criteria:

- circumstantial elements suggesting antemortem exposure to low temperatures, with no rewarming between exposure and death,

- macroscopic and microscopic findings suggesting exposure to low temperatures, according to the available forensic literature,

- postmortem biochemical investigation results consistent with the hypothesis of exposure to low temperatures, according to the available forensic literature (increased urinary catecholamines and metanephrines, increased blood ketones, increased urinary and postmortem serum cortisol),

- exclusion of other causes of death based on all postmortem investigation findings, including negative toxicology.

The second study group (non-hypothermia control group) consisted of 30 forensic autopsies including 15 cases characterized by a short survival time (or short agony, defined as a period of a few seconds up to a few minutes, including 10 cases of witnessed sudden cardiac death with acute coronary thrombosis at postmortem examination and 5 cases of hanging) and 15 cases characterized by a long survival time (or long agony, defined as a period lasting from a few minutes up to hours). This latter subgroup included cases of drug intoxication only.

Non-hypothermia control individuals including traumatic brain injury cases could not be included in the study due to possible CSF contamination with blood.

As stated above, all subjects included in the non-hypothermia control group originated from forensic practice and underwent forensic investigations between 12 and $48 \mathrm{~h}$ after death. The most relevant inclusion criteria consisted of: 
- postmortem serum and CSF availability at autopsy,

- exclusion of traumatic brain injury as the main or contributory cause of death (exclusion of intracranial and intracerebral bleeding, exclusion of cortical contusion, exclusion of cortical and cerebral tissue damage),

- exclusion of hypothermia as the main or contributory cause of death.

Deaths caused by diabetic ketoacidosis, sepsis and liver disease (as the main or contributory causes of death) were excluded in order to avoid central causes of hypothermia and possible interfering laboratory results.

Sample collection

Femoral blood samples were collected prior to autopsy by aspiration with sterile needles and syringes from the femoral vein(s). Femoral blood samples were stored in preservative-free gel and clot activator tubes. These were centrifuged immediately post collection at $3000 \mathrm{~g}$ for $15 \mathrm{~min}$. After centrifugation, the separated supernatant (postmortem serum) was collected and stored in preservative-free tubes. These were transferred to the laboratories immediately post collection.

Undiluted cerebrospinal fluid samples were collected by aspiration using a sterile needle and syringe by suboccipital puncture as soon as possible upon arrival of the bodies at the morgue and prior to autopsy or by aspiration during autopsy (direct intraventricular puncture). No samples were excluded due to blood contamination. All samples were immediately centrifuged at $3000 \mathrm{~g}$ for $15 \mathrm{~min}$. After centrifugation, the separated supernatant was collected and stored in tubes containing sodium fluoride and preservative-free tubes. No specimens were excluded due to insufficient sample volume (minimum accepted volume: $3 \mathrm{ml}$ ). Cerebrospinal fluid samples were transferred to the laboratories immediately post collection.

Laboratory assay

Endocan, S100B, and GFAP concentrations were measured by enzyme-linked immunosorbent assays. NSE concentrations were measured using an electrochemiluminescence immunoassay.

CRP concentrations were measured using an immunoturbidimetric method.

ALP concentrations were determined by standard procedures (International Federation of Clinical Chemistry and Laboratory Medicine reference measurement procedure at $37^{\circ} \mathrm{C}$ ) on a Roche analyser.

Statistical analysis

Data were analysed using GraphPad Prism software 6.0 (published by GraphPad Software, Inc., La Jolla, CA, USA) as a statistical unit. Endocan, ALP, NSE, S100B, GFAP, and CRP concentrations in postmortem serum and CSF in hypothermia-related deaths and non-hypothermia cases were compared nonparametrically. $P$ values less than 0.05 were considered statistically significant. Cut-off levels discriminating hypothermia-related deaths and non-hypothermia cases in both analysed biological samples were not preliminarily identified.

\section{Results}

Tables 1 summarizes the main results pertaining to all studied parameters measured in postmortem serum and CSF in hypothermia and non-hypothermia cases.

Concerning NSE, S100B, and GFAP, the levels measured in CSF were generally higher than the corresponding values in postmortem serum, as already reported by other research teams in the past in situations of both deaths following traumatic brain injury and deaths unrelated to traumatic brain injury. 
Concerning ALP and CRP, conversely, the concentrations observed in postmortem serum were generally more elevated than those found in CSF, in whom a slight activity was nevertheless measured, as already reported by some authors.

Similar endocan values were noticed in both tested biological samples.

Generally, no difference was observed in any of the tested biomarkers in any of the sampled fluid in any of the studied groups.

Analysis of the results obtained from biomarker measurements in postmortem serum and CSF did not reveal statistically significant differences between hypothermia-related deaths and non-hypothermia cases ( $P<0.05$ for all biomarkers in both tested samples in all studied groups).

In addition, in the non-hypothermia group, values measured in both biological samples for each of the analysed markers displayed no significant differences relating to each of the tested subgroups.

Globally considered, these results tend to suggest that, contrary to what is observed in situations of traumatic brain injury, death following exposure to low temperatures is not characterized by significant neuronal, glial, and cerebral endothelial cell injury leading to increased endocan, ALP, NSE, S100B, GFAP, and CRP concentrations in postmortem serum and CSF collected at autopsy.

In other words, the reported results seem to indicate that central nervous system impairment that typifies the hypothermic process does not result in corresponding substantial neuro-pathological and neuro-biochemical changes that might be investigated through specific laboratory analyses in postmortem serum and CSF.

(Table 1)

Table 1 summarizes the main results (ranges and median) of all the studied biomarkers obtained in both the hypothermia and non-hypothermia groups in both tested samples. All abbreviations are mentioned in the text.

\begin{tabular}{|c|c|c|c|c|c|c|}
\hline $\begin{array}{c}\text { Tested biomarker } \\
\begin{array}{c}\text { Studied } \\
\text { group }\end{array}\end{array}$ & $\begin{array}{c}\text { Endocan } \\
(\mathrm{ng} / \mathrm{ml})\end{array}$ & $\begin{array}{c}\text { ALP } \\
(\mathrm{U} / \mathrm{l})\end{array}$ & $\begin{array}{c}\mathrm{NSE} \\
(\mathrm{ng} / \mathrm{ml})\end{array}$ & $\begin{array}{c}\text { S100B } \\
(\mathrm{ng} / \mathrm{ml})\end{array}$ & $\begin{array}{c}\text { GFAP } \\
(\mathrm{ng} / \mathrm{ml})\end{array}$ & $\begin{array}{c}\text { CRP } \\
(\mathrm{mg} / \mathrm{l})\end{array}$ \\
\hline $\begin{array}{c}\text { Hypothermia } \\
\text { postmortem serum } \\
(\mathrm{n}=30)\end{array}$ & $\begin{array}{c}<0.200-0.325 \\
(0.264)\end{array}$ & $\begin{array}{c}170-330 \\
(220)\end{array}$ & $\begin{array}{c}80-250 \\
(150)\end{array}$ & $\begin{array}{c}95-300 \\
(195)\end{array}$ & $\begin{array}{c}0.20-0.50 \\
(0.36)\end{array}$ & $\begin{array}{c}8-15 \\
(9)\end{array}$ \\
\hline $\begin{array}{c}\text { Hypothermia } \\
\text { CSF }\end{array}$ & $\begin{array}{c}<0.200-0.310 \\
(0.271)\end{array}$ & $\begin{array}{c}65-115 \\
(101)\end{array}$ & $\begin{array}{c}800-2300 \\
(1400)\end{array}$ & $\begin{array}{c}130-3100 \\
(560)\end{array}$ & $\begin{array}{c}95-160 \\
(132)\end{array}$ & $3-9$ \\
\hline $\begin{array}{c}\text { Non-hypothermia } \\
\text { postmortem serum } \\
(\mathrm{n}=30)\end{array}$ & $\begin{array}{c}<0.200-0.315 \\
(0.255)\end{array}$ & $\begin{array}{c}185-310 \\
(240)\end{array}$ & $\begin{array}{c}90-240 \\
(160)\end{array}$ & $\begin{array}{c}100-320 \\
(215)\end{array}$ & $\begin{array}{c}0.15-0.55 \\
(0.35)\end{array}$ & $7-18$ \\
\hline $\begin{array}{c}\text { Non-hypothermia } \\
\text { CSF }\end{array}$ & $<0.200-0.325$ & $50-125$ & $900-2100$ & $90-3300$ & $80-155$ & $3-10$ \\
$(\mathrm{n}=30)$ & $(0.267)$ & $(109)$ & $(1510)$ & $(600)$ & $(136)$ & $(6)$ \\
\hline
\end{tabular}

Discussion

Despite the notable progress made over the last few years in the medico-legal field, it is still cumbersome for forensic pathologists to attribute a death to the consequences of exposure to low temperature, whether as a principal cause or as a contributing one (28).

It is still correct to claim a postmortem diagnosis of hypothermia as a "negative" diagnosis, or a diagnosis "of exclusion," which can be formulated, conservatively, only once compatible and 
suggestive circumstantial elements have been obtained and gathered up, on one side, and, on the other side, radiological, morphological, toxicological, and biochemical results have been gathered, which are not contrary to the initial hypothesis (29).

There is no morphological (whether it be radiological, macroscopic, or microscopic) or biochemical (whether it be metabolic, or metabolomic) finding that allows, at least at the current state of knowledge and when considered individually and decontextualized, for a categorical statement that "the death was a consequence of exposure to low temperature." The most established (and most scientifically correct, as could be upheld in court) practice remains, therefore, the collection and combination of as many morphological and biochemical results as possible, which can, when examined as a whole, corroborate, or not contradict, the sole/prevalent/contributing role that exposure to cold has had (or may have had) in the pathogenesis of the death (28-30).

Postmortem morphological data, whether they be radiological (increased aerated lung percentage), macroscopic (frost erythema, Wischnewsky spots within the gastric mucosa), microscopic (ArmanniEbstein kidney) or biochemical (increased urinary catecholamines and metanephrines, increased blood ketones, increased urinary and postmortem serum cortisol, increased free fatty acids in the postmortem serum), do not necessarily indicate with certainty that the death is the result of exposure to low temperatures, even if they may allow one to better direct the diagnosis, especially in cases for which there is suggestive circumstantial evidence, and in the absence of other causes of death $(1,28,29,31-33)$.

In the clinical setting, it has been speculated that cerebral metabolism is reduced by $6-7 \%$ for each degree body temperature decreases. Self-regulation mechanisms within the cerebral blood flow allow for the maintenance of adequate cerebral circulation, even at temperatures of approximately 25 degrees. As temperature decreases beyond a certain point, impairments in cerebral oxygenation can be observed. An insufficient supply of oxygen can seem, for example, to be responsible for the activation of random neurons within those parts of the brain that control vision, resulting in the sensation of seeing "brilliant lights" (2-4).

The effect of lowering temperatures in the brain could, furthermore, be the cause of local injuries on a cellular level (endothelial, neuronal, and glial), with potential occurrences of necrosis and inflammation. On the other hand, even though exposure to low temperatures not seems to be associated with changes in the permeability of the blood-brain barrier, such exposure could be responsible for increases in local vascular permeability nonetheless (5).

Based on the above, the objective of this study was to evaluate whether or not endothelial, neuronal, and glial cell damage, which might occur during the hypothermic process, could also be responsible for changes in concentrations (in the postmortem serum and/or CSF) of molecules normally contained within these cells or expressed within their membranes as a consequence of the occurrence of local necrosis and/or loss of integrity in blood-brain barrier permeability, and if these changes could be investigated by postmortem biochemical analyses.

Preliminary results, as shown in Table 1 , have not allowed for the identification of a specific biochemical profile for death by hypothermia in the postmortem serum and/or the CSF. The measured concentrations of all the tested molecules have failed to reveal statistically significant differences between hypothermic and non-hypothermic cases in either the postmortem serum or CSF.

In respect to other studies conducted within the field of forensics, which have revealed how CSF S100B and NSE concentrations are increased in cases of antemortem traumatic brain injuries, as compared to control cases without traumatic brain injuries, the negative results obtained through this study are probably due to the absence of "major" traumatic brain injuries (in the parenchyma and in the cerebrovascular structures) in the cases of lethal hypothermia considered.

It could be speculated that, in these cases, the hypothermic process within the brain most likely occurs in the form of widespread hypoxic damage that gradually happens and not in the form of significant localized injury, hence lacking, based on the obtained results, significant neuro-pathological changes objectifiable from a biochemical perspective.

This appears to be a legitimate speculation when one considers the meaning of therapeutic hypothermia as a protective measure for the brain in the clinical setting. 
Nevertheless, the reported data should not appear to be discouraging, and should, rather, be considered a first attempt at defining a cerebral "biochemical" profile of deaths due to hypothermia. The hypothesis concerning the existence of neuro-pathological changes, potentially capable of being examined through forensic biochemical analyses, remains, in our opinion, both enticing and legitimate, taking into consideration the typical neurological symptoms of hypothermia.

Therefore, a "panel" of brain damage biomarkers different and alternative must still be defined, in order to identify molecules stable during the postmortem period, indicative of a diffuse hypoxic brain injury, and with changes in their concentrations in the postmortem serum and/or CSF, clearly distinguishable from those potentially measurable in cases of death unrelated to both cold exposure and antemortem traumatic brain injury.

\section{References}

1. Rousseau G, Reynier P, Jousset N, et al. Updated review of postmortem biochemical exploration of hypothermia with a presentation of standard strategy of sampling and analyses. Clin Chem Lab Med CCLM. 2018;56:1819-1827.

2. Mallet ML. Pathophysiology of accidental hypothermia. QJM Int J Med. 2002;95:775-785.

3. Castellani JW, Young AJ. Human physiological responses to cold exposure: Acute responses and acclimatization to prolonged exposure. Auton Neurosci. 2016;196:63-74.

4. Jeican II. The pathophysiological mechanisms of the onset of death through accidental hypothermia and the presentation of "The little match girl" case. Clujul Med. 2014;87:54-60.

5. Oehmichen M, Auer RN, König HG. Forensic Neuropathology and Associated Neurology. Berlin Heidelberg: Springer-Verlag; 2006.

6. Liu Y, Liang X, Xu X, et al. Increased Serum Alkaline Phosphatase in Patients with Acute Ischemic Stroke. J Stroke Cerebrovasc Dis. 2018.

7. Sahu S, Nag DS, Swain A, et al. Biochemical changes in the injured brain. World J Biol Chem. 2017;8:21-31.

8. Lorente L. Biomarkers associated with the outcome of traumatic brain injury patients. Brain Sci. 2017;7.

9. Pleines UE, Morganti-Kossmann MC, Rancan M, et al. S-100 $\beta$ reflects the extent of injury and outcome, whereas neuronal specific enolase is a better Indicator of neuroinflammation in Patients With Severe Traumatic Brain Injury. J Neurotrauma. 2001;18:491-498.

10. Osuna E, Perez-Carceles MD, Luna A, et al. Efficacy of cerebro-spinal fluid biochemistry in the diagnosis of brain insult. Forensic Sci Int. 1992;52:193-198.

11. Nogami M, Takatsu A, Endo N, et al. Immunohistochemistry of neuron-specific enolase in neurons of the medulla oblongata from human autopsies. Acta Histochem. 1998;100:371-382.

12. Nogami M, Takatsu A, Ishiyama I. Immunohistochemical study of neuron-specific enolase in human brains from forensic autopsies. Forensic Sci Int. 1998;94:97-109.

13. Faa A, Senes G, Locci A, et al. S100B protein expression in the heart of deceased individuals by overdose: a new forensic marker? Clinics. 2012;67:821-826.

14. Vázquez MD, Sánchez-Rodriguez F, Osuna E, et al. Creatine kinase BB and neuron-specific enolase in cerebrospinal fluid in the diagnosis of brain insult. Am J Forensic Med Pathol.

1995;16:210- 214. 
15. Ondruschka B, Sieber $\mathrm{M}$, Kirsten $\mathrm{H}$, et al. Measurement of cerebral biomarkers proving traumatic brain injuries in post-mortem body fluids. J Neurotrauma. 2018;35):2044-2055.

16. Ondruschka B, Pohlers D, Sommer G, et al. S100B and NSE as Useful Postmortem Biochemical Markers of Traumatic Brain Injury in Autopsy Cases. J Neurotrauma. 2013;30:1862-1871.

17. Gos T, Schroeter ML, Lessel W, et al. S100B-immunopositive astrocytes and oligodendrocytes in the hippocampus are differentially afflicted in unipolar and bipolar depression: A postmortem study. J Psychiatr Res. 2013;47:1694-1699.

18. Sieber M, Dreßler J, Franke $\mathrm{H}$, et al. Post-mortem biochemistry of NSE and S100B: A supplemental tool for detecting a lethal traumatic brain injury? J Forensic Leg Med. 2018;55:65-73.

19. Li D-R, Zhu B-L, Ishikawa T, et al. Postmortem serum protein S100B levels with regard to the cause of death involving brain damage in medicolegal autopsy cases. Leg Med. 2006;8:71-77.

20. Li D-R, Michiue T, Zhu B-L, et al. Evaluation of postmortem S100B levels in the cerebrospinal fluid with regard to the cause of death in medicolegal autopsy. Leg Med. 2009;11:S273-S275.

21. Benjamin Ondruschka, Sandra Schuch, Monique Sieber, et al. Biochemistry in traumatic brain injury: Even useful in postmortem setting. Rom J Leg Med. 2017;25:301-302

22. Scherpereel A, Depontieu F, Grigoriu B, et al. Endocan, a new endothelial marker in human sepsis. Crit Care Med. 2006;34:532-537.

23. Isgrò MA, Bottoni P, Scatena R. Neuron-specific enolase as a biomarker: biochemical and clinical aspects. In: Scatena R. Advances in Cancer Biomarkers: From biochemistry to clinic for a critical revision. Dordrecht: Springer Netherlands; 2015. p. 125-143.

24. Kaiser E, Kuzmits R, Pregant $P$, et al. Clinical biochemistry of neuron specific enolase. Clin Chim Acta. 1989;183:13-31.

25. Schiff L, Hadker $\mathrm{N}$, Weiser $\mathrm{S}$, et al. A literature review of the feasibility of glial fibrillary acidic protein as a biomarker for stroke and traumatic brain injury. Mol Diagn Ther. 2012;16:79-92.

26. Kinoshita A, Onoda H, Imai N, et al. C-Reactive Protein as a Prognostic Marker in Patients with Hepatocellular Carcinoma. Hepatogastroenterology. 2015;62:966-970.

27. Watt DG, Horgan PG, McMillan DC. Routine clinical markers of the magnitude of the systemic inflammatory response after elective operation: A systematic review. Surgery. 2015;157:362-380.

28. Turk EE. Hypothermia. Forensic Sci Med Pathol. 2010;6:106-115.

29. Palmiere C, Teresiński G, Hejna P. Postmortem diagnosis of hypothermia. Int J Legal Med. 2014;128:607-614.

30. Rousseau G, Chao de la Barca JM, Rougé-Maillart C, et al. A serum metabolomics signature of hypothermia fatalities involving arginase activity, tryptophan content, and phosphatidylcholine saturation. Int J Legal Med 2018 doi: 10.1007/s00414-018-1937-y.

31. Michiue T, Sakurai T, Ishikawa T, et al. Quantitative analysis of pulmonary pathophysiology using postmortem computed tomography with regard to the cause of death. Forensic Sci Int. 2012;220:232-238.

32. Kawasumi Y, Onozuka N, Kakizaki A, et al. Hypothermic death: Possibility of diagnosis by postmortem computed tomography. Eur J Radiol. 2013;82:361-365. 
33. Hyodoh $\mathrm{H}$, Watanabe $\mathrm{S}$, Katada $\mathrm{R}$, et al. Postmortem computed tomography lung findings in fatal of hypothermia. Forensic Sci Int. 2013;231:190-194. 